\title{
Networking
}

\section{Reciprocity and paying attention to process: an important issue for the UK Clinical Research Network in primary care?}

\begin{abstract}
Jo Cooke Director, Trent Research \& Development Support Unit, School for Health and Related Research (ScHARR), University of Sheffield, Sheffield, UK, Nigel Mathers Professor of General Practice and Unit Director, Academic Unit of Primary Medical Care, University of Sheffield, Sheffield, UK and Caroline Mitchell Senior Lecturer/General Practitioner, Academic Unit of Primary Medical Care, University of Sheffield, Sheffield, UK
\end{abstract}

\section{Introduction}

The Research \& Development (R\&D) strategy for health in the UK (Department of Health, 2006) has established an infrastructure of research networks, developed under the auspices of the UK Clinical Research Collaboration (UK CRC) and the National Institute for Health Research (NIHR), which, it is anticipated, will produce an efficient and effective interface between the NHS and academia to enable highquality clinical research. Such networks, among other things, aim to produce research for practice by supporting recruitment to clinical trials. This paper suggests some problems in relation to assumptions in this strategy, which imply that once networks have been established, the interface with practice is somehow 'sorted'. It explores particular issues for the primary care practiceresearch interface, where recruitment and capacity building have experienced significant barriers and difficulties in the past. The paper pays particular attention to valuing 'close to practice' principles in relation to the effectiveness of networks, and their ability to build capacity, and highlights the importance of process issues in achieving network aims. The paper highlights the experience and learning of existing networks in primary care, and evidence about what supports recruitment to clinical trials. The current lack of emphasis on

\footnotetext{
Address for correspondence: Jo Cooke, Trent RDSU, School of Health and Related Research, ICOSS, University of Sheffield, 219 Portobello, Sheffield S1 4DP, UK. Email: j.m.cooke@sheffield.ac.uk
}

developing research skills and leadership in frontline practitioners is also considered, and specific concerns regarding reciprocity between research and practice partnerships are highlighted. The need for development of such skills in non-clinical researchers is also important but not the subject of this paper. Concern is expressed that if these issues, and others related to the social capital of networks, are not addressed, then the UK Clinical Research Network (UKCRN) will not be able to deliver on its objectives.

\section{Background}

There has been considerable investment in developing research networks in primary care internationally (Griffiths et al., 2000; Van Weel et al., 2000; Farmer and Weston, 2002; North American Primary Care Research Group Committee on Building Research Capacity and the Academic Family Medicine Organisations Research Sub-Committee, 2002; Lindbloom et al., 2004). The emphasis and purpose of Primary Care Research Networks (PCRNs) vary from maximising participation in research to developing a culture of producing research within practice. Many authors argue that networks have a function to move practitioners from participation to becoming active researchers in their own right (Clement et al., 2000; Ryan and Wyke, 2001; Farmer and Weston, 2002).

In the last decade, the number of PCRNs in the UK expanded based on recommendations from 
the Mant report (NHS Executive, 1997), which stressed the need to develop research for, with and by primary care practitioners. The National Coordinating Centre for Research Capacity Development funded 20 PCRNs within their programme of work to increase research capacity defined as 'a process of individual and institutional development which leads to higher levels of skills and greater ability to perform useful research' (Trostle, 1992, p. 1321).

The importance of networks in the prosecution of research per se should not be underestimated. Research for practice can be undertaken through using evidence gathered from practice (for example, using electronic data to undertake epidemiological research), and can assist research with practitioners by promoting active collaborations. Engaging practitioners to help with recruitment for large projects could fit into this category, and networks have been found to be important in accessing representative populations in primary care in this regard (Hammersley et al., 2002). In addition, by adopting a learning and participatory approach, networks can enable research by individual practitioners and practice teams to grow 'bottom-up' research ideas.

Over the past year there has been initiation of disease-specific networks, comprehensive networks and a national structure of PCRNs. In these developments, however, the concept of developing capacity seems to have lost momentum and emphasis, and has been replaced by notions of recruitment and 'participation'. In a recent discussion document (Department of Health, 2006), networks are recognised as essential to the proposed strategy; however, capacity development for practitioners is no longer a core network function. The purpose of networks is described as follows: increasing participation through maximising recruitment, integrating research and patient care; increasing the quality, speed and coordination of clinical research; and providing an explicit means by which the NHS can meet the health research needs of the industry. While these aims are understandable they may well miss out on essential elements of linking and developing research that is close to practice, in nurturing the important synergies between practice and research, and in developing the research capacity of individuals and teams within the networks.
Recent literature points to a history of difficulties and poor working practices between researchers and primary care. For example, McKinley et al. (2002, p. 971) state:

practitioners can feel they are regarded as mere conduits to reservoirs of people on their lists by researchers who have paid scant regard to practitioners preferences about study designs and recruitment strategies.

Many of the networks that have been developed in primary care up to this point have been sensitive to this view, and have been shaped around trust and social capital (Fenton et al., 2001), using methods that include 'bottom-up' and whole systems approaches to involve practitioners as leaders and 'doers' of research, as well as collaborators and patient recruiters (Thomas and While, 2001; Thomas et al., 2001). Other authors have focussed on the importance of process in the evaluation of these networks and suggest that such process issues are essential in building a body of understanding about what works in producing useful research (Griffiths et al., 2000; Fenton et al., 2001). Capacity building can not only support incentives for practitioners to participate in networks but also help to support and build connections to show how research can be beneficial to practice and to patients (Thomas et al., 2001).

\section{Applying principles of capacity building to shape the conduct of research networks}

The principles of research capacity building are useful in mapping some of the process issues which could inform a code of conduct for networks, and which may also contribute to their productivity. These principles can inform appropriate approaches to undertaking research with practitioners, avoiding the difficulties highlighted by McKinley and colleagues, and can ensure elements of ownership, reciprocity and incentives.

Trent Research and Development Support Unit (RDSU) has developed some principles of research capacity building through experience of supporting research alongside practice, and the use of research evidence (Cooke, 2005). These are listed in Box 1. A number of these issues are 


\section{Box 1 Principles of Research Capacity Building}

\section{Principle}

Building skills and confidence

Developing research close to practice

Supporting research linkages, partnerships and collaborations

Ensuring appropriate dissemination

Building continuity and sustainability

Building infrastructure to support research

\section{Description of principle}

Building research skills and confidence through training, supervision and mentorship, and by applying skills to practice

Developing research ideas through developing ideas pertinent to practice issues, by working with practitioners, service users and policy makers to make research relevant and immediately useful, as well as maximising interest, ownership and participation

Developing linkages and partnerships through developing collaborations between research and practice, service users, industry, communities and researchers and practitioners in other countries

Developing dissemination methods that ensure quality (through peer reviewed publications and conference presentations), and impact (through diverse and innovative methods)

Recognising and utilising skills that have been developed. Developing further opportunities for research activity through applications for further funding, and career development options (fellowships etc)

Developing structures and processes to ensure the smooth and effective running of projects, including project management techniques, use of protocols and support staff

Note: these principles have been developed through the work of Trent RSDU based on the framework by Cooke (2005).

fundamental to the new networks, but some may be at risk as discussed above. In particular, it is the close to practice principle that appears marginalised, but which is important, not only for the effective running of research projects but also when the impact of research on primary care practice is considered.

\section{Closeness to practice: an important principle for productivity, recruitment and impact}

Research, which is conducted 'close' to practice, is useful for two reasons. Firstly, in working closely with practitioners during the development and conduct of research projects, they are more likely to reach a satisfactory conclusion, and be clinically appropriate (Parry et al., 2003). A systematic review addressing barriers to participation in randomised controlled trials (RCTs) (Prescott et al., 1999) has highlighted some key issues for working close to practice. The review highlights that barriers to practitioner participation include:

- fears around the impact on the doctor-patient relationship;

- concern for patients as they may not receive the treatment that turned out to be the best;

- the perception and importance of, and interest in, the research topic;

- incompatibility of the research protocol with normal practice;

- time constraints;

- training.

Most of the studies included in the review were based in secondary care, but many of the barriers are valid, and are often compounded, in primary 
care contexts. For example, the relationship with patients in primary care is not only for a single treatment period but generally for a long period of time. Challenges to the quality and rapport in the practitioner-patient relationship, perhaps as a result of research activity, will therefore have a longer-term impact. Prescott et al. (1999) and others reviewing RCTs in primary care (Foy et al., 2003) have come to similar conclusions about methods of improving the effectiveness of recruitment strategies to inform the conduct of networks. These include working closely with practitioners who have a role in developing priorities, shaping research ideas and informing methodologies that are more pragmatic in nature. Empirical evidence suggests that practitioners are more likely to engage in research if they see its relevance to their own practice, and developing research protocols with practitioners can ensure that research work blends better with practice work, and will pay attention to time constraints. Training and dialogue with practitioners in networks can develop research skills, and will help to 'unpack' ethical issues and research-practice dilemmas (Foy et al., 2003). PCRNs established following the publication of the Mant Report (NHS Executive, 1997) developed close relationships with local RDSUs. Developing strong links between the networks and RDSUs is important here, as they may well have a role to play in assisting in these process issues: in supporting training within networks, priority setting and protocol development at the practice-research interface.

Secondly, developing research close to practice can mean results are more likely to be taken up in practice and have some impact (National Audit Office, 2003). Networks may help overcome the translational block of integrating new knowledge into practice (Lindbloom et al., 2004).

\section{Close to practice: keeping a focus on research capacity building in practitioners}

Some of the key literature around generating knowledge for primary care practice highlights the need for developing research practitioners (Farmer and Weston, 2002; North American Primary Care Research Group Committee on
Building Research Capacity and the Academic Family Medicine Organisations Research SubCommittee, 2002). Examples are given of how networks can help build capacity in primary care practitioners, by developing skills and knowledge in research, and by nurturing interest and providing opportunities and research experience (Thomas and While, 2001; Macfarlane et al., 2005). Practitioner-led research activity has been promoted through a variety of mechanisms in PCRNs, including a whole systems approach where research is shaped, and led by network members (Thomas and While, 2001), by funding pilot work through bursary schemes supporting external grant submissions by network members (Pitkethly and Sullivan, 2003), and through other approaches such as designated research teams. The notion that networks may provide opportunities as a 'way into' research is an important concern in building a primary care academic workforce. The lack of focus on this issue in the new networks is of concern. Not all primary care practitioners want to be research leaders, and many are happy to contribute to the research endeavour by engaging in research at a minimum level of participation; however, others may welcome research opportunities that can act as an incentive. Financial incentives have been well documented as an important consideration in recruitment (Foy et al., 1998), but in our experience this it is not the full story. Issues of professional development, professional interest, benefits to patients, the quality of services and professional status also need to be considered.

\section{Conclusions}

This paper has highlighted some of the key process issues that are important in the development and future conduct of the networks under the direction of the UKCRN. The importance of process issues in how the networks should operate has been stressed, and we suggest that adopting research capacity building principles, in particular that of working closely with practice, should shape a code of network conduct and perhaps form the basis of accreditation, which will facilitate their productivity. Strong links with RDSUs are also stressed to maximise expertise that currently exists in this regard. Concern is expressed 
about the lack of continuing opportunity to build research capacity in the health care workforce in the new UKCRN networks. The current emphasis on recruitment and participation may well have the unintended consequences of reducing the infrastructure and potential of academic primary care, and research productivity in the future.

\section{Declaration}

The authors have no competing interests.

\section{References}

Clement, S., Pickering, A., Rowlands, G., Krishnan, T., Candy, B. and De Lusignan, S. 2000: Towards a conceptual framework for evaluating primary care research networks. British Journal of General Practice 50, 651-52.

Cooke, J. 2005: A framework to evaluate research capacity building in health care. BMC Family Practice 6(44). Retrieved from http://www.biomedcentral.com/1471-2296/ $6 / 44$.

Department of Health. 2006: Best research for best health: a new national health research strategy. London: Department of Health.

Farmer, E. and Weston, K. 2002: A conceptual model for capacity building in Australian primary health care research. Australian Family Physician 31, 1139-142.

Fenton, F., Harvey, J., Griffiths, F., Wild, A. and Sturt, J. 2001: Reflections from organization science of primary health care networks. Family Practice 18, 540-44.

Foy, R., Parry, J. and McAvoy, B. 1998: Clinical trials in primary care. British Medical Journal 317, 1168-169.

Foy, R., Parry, J., Duggan, A., Delaney, B., Wilson, S., Lewin-van den Broek, N., Lassen, A., Vickers, L. and Myres, P. 2003: How evidence based are recruitment strategies to randomized controlled trials in primary care? Experience from seven studies. Family Practice 20, 83-92.

Griffiths, F., Wild, A., Harvey, J. and Fenton, E. 2000: The productivity of primary care research networks. British Journal of General Practice 50, 913-15.

Hammersley, V., Hippersley-Cox, J., Wilson, A. and Pringle, M. 2002: A comparison of research general practices and their patients with other practices - a cross-sectional survey in Trent. British Journal of General Practice 52, 463-68.
Lindbloom, E.J., Wewigman, B.G. and Hicker, J.M. 2004: Practice-based research networks. The laboratories of primary care research. Medical Care 42, Supplement III45-49.

Macfarlane, F., Shaw, S., Greenhalgh, T. and Carter, Y.H. 2005: General practices as emergent research organizations: a qualitative study into organizational development. Family Practice 22, 298-304.

McKinley, R.K., Dixon-Woods, M. and Thornton, H. 2002: Participating in primary care research. British Journal of General Practice 52, 971-72.

National Audit Office. 2003: An international review on Governments' research procurement strategies. A paper in support of getting the evidence: using research in policy making. London: The Stationery Office.

NHS Executive. 1997. National working party on R\&D in primary care. Final report (Chair: Professor D. Mant). Leeds: NHS Executive.

North American Primary Care Research Group Committee on Building Research Capacity and the Academic Family Medicine Organisations Research Sub-Committee. 2002: What does it mean to build research capacity? Family Medicine 34, 678-84.

Pitkethly, M. and Sullivan, F. 2003: Four years of TayRen, a primary care research and development network. Primary Care Research and Development 4, 279-83.

Prescott, R.J., Counsell, C.E., Gillespie, W.J., Grant, A.M., Russell, I.T., Kiauka, S., Colthart, I.R., Ross, S., Shepherd, S.M. and Russell, D. 1999: Factors that limit the quality, number, and progress of randomised control trials. Health Technology Assessment Programme 3. Retrieved from http://www.hta.nhsweb.nhs.uk/execsumm/summ320.htm.

Ryan, K. and Wyke, S. 2001: The evaluation of Primary Care Research Networks in Scotland. British Journal of General Practice 51, 154-55.

Thomas, P., Griffiths, F., Kai, J. and O'Dwyer, A. 2001: Networks for research in primary care. British Medical Journal 322, 588-90.

Thomas, P. and While, A. 2001: Increasing research capacity and changing the culture of primary care towards reflective inquiring practice: the experience of West London Research Network (WeLReN). Journal of Interprofessional Care 15, 133-39.

Trostle, J. 1992: Research capacity building and international health: definitions, evaluations and strategies for success. Social Science \& Medicine 35, 1321-324.

Van Weel, C., Smith, H. and Beasley, J.W. 2000: Family practice research networks: experience from three countries. The Journal of Family Practice 49, 938-43. 